\title{
Chromosome Behaviour at Meiosis in Saccharomyces cerevisiae
}

\author{
BY HIDEO TAMAKI \\ Doshisha Women's College Kyoyo, Japan
}

(Received 24: March 1965)

\begin{abstract}
SUMMARY
The behaviour of the chromosomes of diploid strains of Saccharomyces cerevisiae was studied in organisms which had been treated with snail digestive juice after fixation, and then stained with the HCl-Giemsa stain. Coagulation of chromatin was observed after late prophase-I in some strains and for this reason the chromosome number of these strains could not be determined. One diploid strain (H $102 \times \mathrm{H}_{9}$ ) showed no chromatin coagulation, and was shown to have eighteen bivalents at metaphase-I.
\end{abstract}

\section{INTRODUCTION}

Since the investigations of van Leeuwenhoek, cytological observations of nuclear behaviour in yeast have been made by many cytologists, but the details of the division process and the chromosome number have not yet been clarified in mitosis or in meiosis. Drs R. K. Mortimer and D. C. Hawthorne have genetic evidence indicating that Saccharomyces cerevisiae has a minimum of fifteen linkage groups (personal communication). In the present study an attempt has been made to determine the chromosome number of $\boldsymbol{S}$. cerevisiae by direct cytological methods, and to study the movement of the chromosome at meiosis in diploid strains.

\section{METHODS}

Organisms. The following strains of Saccharomyces cerevisiae were used in the present work: н 9 , mating type a, histidine requirer $\left(\mathrm{hi}_{2}\right) ; \mathrm{H} 11$, mating type $\alpha$, histidine and uracil requirer $\left(\mathrm{hi}_{2}, \mathrm{ur}_{1}\right)$; н 102 mating type $\alpha$, adenine and tryptophan requirer $\left(\mathrm{ad}_{6}, \mathrm{tr}_{5}\right)$; н 104, mating type a, adenine, arginine and tryptophan requirer $\left(\operatorname{ad}_{6}, \operatorname{ar}_{4}, \operatorname{tr}_{5}\right) ; \mathrm{H} 203$, mating type $\alpha ; \mathrm{H} \mathrm{204}$, mating type a.

Diploid strains were obtained from the following crosses: $\mathrm{H} 11 \times \mathrm{H} 104$; $\mathrm{H} 203 \times \mathrm{H}$ 204; н $102 \times$ н 9 . These crosses were made by the minimal plate mating technique of Takahashi, Saito \& Ikeda (1958) or the mass mating technique of Lindegren \& Lindegren (1943). The genotype of each diploid strain was confirmed by tetrad analysis.

Culture media. The complete culture medium had the following composition (g./1.); peptone, 10; Difco yeast extract, 10; glucose, 20. Spore-forming cultures of yeast were maintained in the sodium acetate medium of Fowell (1952) or the potassium acetate medium of McClary, Nulty \& Miller (1959). Complete medium agar and sodium acetate or potassium acetate sporulation medium agar contained Bacto-agar $20 \mathrm{~g}$./l. 


\section{Cytological procedures}

Organisms were prepared for cytological observation by the following procedure.

1. When grown in liquid complete medium at $28^{\circ}$ for $48 \mathrm{hr}$ they were transferred to liquid sporulation medium; when they were grown on solid complete medium at $28^{\circ}$ for $48 \mathrm{hr}$ they were transferred to solid sporulation medium.

2. Organisms were collected either by centrifugation of liquid cultures, or by scraping growth from the surface of solid media with a platinum needle; they were fixed in Flemming's strong fluid for $24 \mathrm{hr}$ in a cold dark room. The fixed materials were washed five times with distilled water by repeated centrifugations, and material which showed a darker appearance was bleached in $\mathrm{B} \% \mathrm{H}_{2} \mathrm{O}_{2}$ solution and washed twice as before.

3. The next step was treatment with snail digestive juice at $28^{\circ}$ for $30 \mathrm{~min}$., washing off the residue by three successive centrifugations in distilled water. This facilitated the subsequent smearing of the fixed cell materials. The juice was got from snails which had not been fed for 2 days, and it was sterilized by antibiotics without dilution.

4. The materials were hydrolyzed with $\mathrm{N}-\mathrm{HCl}$ at $60^{\circ}$ for $16 \mathrm{~min}$., washed three times as before, and the final water suspension spread on clean cover glasses and dried at room temperature.

5. The material which adhered to the cover glass was stained with diluted Giemsa solution (1 vol. Giemsa stock solution diluted with 80 vol. 0.02 M-phosphate buffer, $\mathrm{pH} 6.8$ ) at $28^{\circ}$ for $7 \mathrm{~min}$. and washed with the same buffer solution. The stained material was then mounted and squashed.

In addition to the Giemsa stain, the Feulgen stain and methyl green (1\%) were also used. The results obtained were the same, i.e. the same bodies (chromatin in meiosis) were well stained. The Giemsa stain was preferred for further study, since it gave somewhat better staining.

\section{RESULTS}

\section{Nuclear behaviour in meiosis of Saccharomyces cerevisiae diploid strain $\mathrm{H} 11 \times \mathrm{H} 104$}

The Saccharomyces cerevisiae diploid strain $\mathrm{H} 11 \times \mathrm{H} 104$, was grown in liquid sporulation medium with aeration at $28^{\circ}$ and samples prepared every $24 \mathrm{hr}$ as described in Methods. Various shapes were observed in each sample; for convenience, these have been divided into seven stages as shown Pl. 1, figs. A-G. The frequencies of appearance of each stage after different times of incubation are shown in Table 1. Stage A cells and possibly some of the stage $\mathbf{E}$ cells will sporulate; $\mathbf{B}, \mathbf{C}$ and $\mathrm{D}$ are intermediate stages in sporulation. Stage $\mathrm{F}$ shows tetrad formed at completion of sporulation.

The coagulation of chromatin observed in this strain in liquid and solid sporulation cultures, was also observed in the diploid strain high chromatin coagulation, it was not possible to determine the chromasome number from these figures. 


\section{Meiosis of Saccharomyces cerevisiae diploid strain ${ }_{\mathrm{H}} 102 \times{ }_{\mathrm{H}} 9$}

As shown in Table 1, after Saccharomyces cerevisiae strain H $11 \times$ H 104 had been kept for $24 \mathrm{hr}$ on sporulation medium, metaphase I was observed frequently, but no tetrads. After $48 \mathrm{hr}$, however, all the stages of meiosis were observed. Accordingly, the $S$. cerevisiae diploid strain $\mathrm{H} 102 \times \mathrm{H} 9$ was incubated on a solid complete medium at $28^{\circ}$ for $48 \mathrm{hr}$, then transplanted to solid sporulation medium and fixed after incubation for $48 \mathrm{hr}$. Plate 2, figs. A-C, shows three stages of the prophase of meiosis I in strain $\mathrm{H} 102 \times \mathrm{H}$. In the case of strains $\mathrm{H} 11 \times 104$ or $\mathrm{H} 203 \times \mathrm{H} 204$,

Table 1. Frequency of meiotic stages at spore formation in Saccharomyces cerevisiae cross $\mathrm{H} 11 \times \mathrm{H} 104$

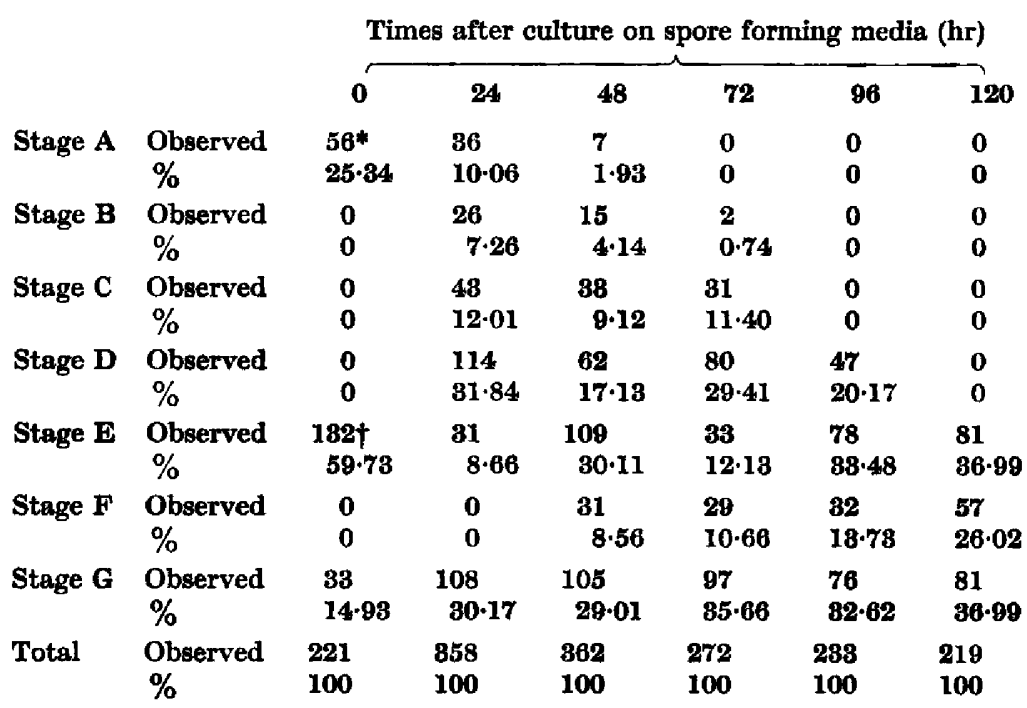

Stages A-G correspond with those of Pl. 1, figs. A-G, respectively. * Mitotic figure. + Stage of Pl. 1, Fig. H.

some of the chromatin showed coagulation as well as heteropycnosis in late prophase. But in the case of strain $\mathrm{B} 102 \times \mathrm{H} 9$, no coagulation into chromatin bodies was observed. Plate 2, fig. C respresents diakinesis and Pl. 2, fig. $\mathrm{J}$ is a diagrammatic sketch of the chromosomes of Pl, 2, fig. C. Plate 2, fig. D shows metaphase I with eighteen bivalents; one of twelve cells examined which showed essentially the same number of bivalents; fig. $K$ is a sketch of fig. $D$. Plate 2, fig. $E$ may be taken as anaphase $I$, since the chromosomes appear to be separated into two poles. The next expected stage should be a diad stage, but no such stage was seen. Plate 2, fig. F shows what may be a stage of metaphase II: 36 chromosomes were easily counted (see sketch, fig. L). Plate 2, fig. G shows anaphase II and 58 chromosomes were counted. The number of chromosomes in anaphase II ought to be 72, according to the number found at metaphase I (Pl. 2, fig. D), but some of the chromosomes might have been pushed out of the cell by the squash method used. The following stage should be the distribution of these chromosomes of four daughter nuclei (PI. 2, fig. H), with subsequent formation of a discrete tetrad (Pl. 2, fig. I). 
The strains used in this study seem to be normal diploids. Polyploidy would be expected to be detected through irregular segregation of genetic markers (Roman, Phillips \& Sands 1955, Mortimer, 1958). The results of tetrad analysis of 32 asci showed decreased spore viability (Table 2). The decreased viability suggests polypolyploidy or polysomy, but the segregation ratios are consistent only with this strain being diploid. More extensive analysis with markers for each linkage group should be undertaken to settle this point.

Table 2. Segregation of three markers among ascospores of Saccharomyces cerevisiae cross H $_{102} \times \mathrm{H} 9$ diploid strain. Only asci containing four spores were dissected

Segregational ratio

Ascus

8

12

3
5
10
14
19

1

9

13

18

21

23

24.

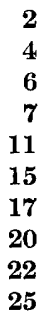

27

28

29

30

31

32

Total

$\overbrace{\text { AD:ad HI:hi TR:tr }}^{\text {Th }}$

4 viable spores

$\begin{array}{lll}2: 2 & 2: 2 & 2: 2 \\ 2: 2 & 2: 2 & 2: 2 \\ 2: 2 & 2: 2 & 2: 2\end{array}$

3 viable spores

$\begin{array}{lll}2: 1 & 1: 2 & 2: 1 \\ 1: 2 & 1: 2 & 2: 1 \\ 2: 1 & 1: 2 & 1: 2 \\ 1: 2 & 2: 1 & 1: 2 \\ 1: 2 & 1: 2 & 1: 2\end{array}$

2 viable spores

$\begin{array}{lll}2: 0 & 1: 1 & 1: 1 \\ 1: 1 & 1: 1 & 1: 1 \\ 2: 0 & 2: 0 & 1: 1 \\ 1: 1 & 0: 2 & 2: 0 \\ 1: 1 & 1: 1 & 2: 0 \\ 0: 2 & 1: 1 & 1: 1 \\ 2: 0 & 2: 0 & 1: 1 \\ 1: 1 & 1: 1 & 0: 2\end{array}$

1 viable spore

$\begin{array}{lll}1: 0 & 0: 1 & 0: 1 \\ 1: 0 & 1: 0 & 1: 0 \\ 1: 0 & 0: 1 & 0: 1 \\ 0: 1 & 0: 1 & 0: 1 \\ 1: 0 & 1: 0 & 0: 1 \\ 0: 1 & 1: 0 & 1: 0 \\ 1: 0 & 0: 1 & 0: 1 \\ 1: 0 & 0: 1 & 0: 1 \\ 0: 1 & 1: 0 & 1: 0 \\ 0: 1 & 1: 0 & 0: 1\end{array}$

0 viable spore

$\begin{array}{ccc}0: 0 & 0: 0 & 0: 0 \\ 0: 0 & 0: 0 & 0: 0 \\ 0: 0 & 0: 0 & 0: 0 \\ 0: 0 & 0: 0 & 0: 0 \\ 0: 0 & 0: 0 & 0: 0 \\ 0: 0 & 0: 0 & 0: 0\end{array}$

29:24 26:27 25:28

$$
\begin{aligned}
& \chi^{2}=0.472 \quad \chi^{2}=0.008 \quad \chi^{2}=0 \cdot 170 \\
& 0.5<P<0.750 .95<P<0.990 .75<P<0.9
\end{aligned}
$$




\section{DISCUSSION}

Many observations have been made by several investigators about the chromosome number in yeast, but the numbers reported hitherto have varied considerably and no definite conclusion has been reached. For example, Swellengrabel (1905), Fuhrmann (1906), Badian (1937), Shinoto \& Yuasa (1941), and DeLamater (1950) reported that the diploid chromosome number in a presumably diploid yeast was four. Levan (1947) reported at least ten chromosomes in the mycelial type of a brewery yeast cell after camphor treatment. Leitz (1951) found three chromosomes in the haploid and six in the diploid stages of Zygosaccharomyces priorianus. Subramaniam (1946) presented evidence for what he considered to be two chromosomes in a brewery yeast, but subsequently, Prahlada Rao \& Subramaniam(1952) found four, six and eight chromosomes in this organism, and concluded that these variations were due to 'endopolyploidy'. After examining many diploid, triploid and tetraploid cells of a strain of Saccharomyces, McClary, Williams, Lindegren \& Ogur (1957) came to the conclusion that the haploid chromosome number is four.

All these demonstrations were made by using certain squash methods or preparations which did not yield satisfactory mitotic and meiotic figures. Also some of the difficulties may be attributed to unusual behaviour of the strains used by many of these investigators; i.e. some strains showed very high incidence of chromatin coagulation throughout the mitotic or meiotic cycles similar to that shown by our strain $S$. cerevisiae $\mathrm{H} 11 \times \mathrm{H}$ 104. It is not known whether this coagulation results from endopolyploidy, as postulated by Subramaniam, or from other causes.

The success of the present studies is the result of the use of snail digestive juice, which facilitated the spreading of cells and chromosomes into one plane. For this reason, the normal orientation of the chromosomes during the different stages of meiosis was disturbed. A similar attempt was made by Yuasa (1960) with a lytic enzyme preparation from Streptomyces, and which was also capable of digesting yeast cell walls. However, the enzyme used by Yuasa was not helpful in discerning individual chromosomes. The present observation is well supported by recent genetic studies of Drs R. K. Mortimer and D. C. Hawthorne (personal communication). They have obtained evidence for at least fifteen linkage groups based on independently segregating centromeres and, in addition, have identified three more groups of genes that are not yet associated with any of the established linkage groups.

The support of the Research Grant of Doshisha Women's College for this project is gratefully acknowledged. The author wishes to thank Drs E. Balbinder and R. K. Mortimer for valuable help in the preparation of the manuscript.

\section{REFERENCES}

Badian, J. (1937). Sur la cytologie des levures. Bull. intern. acad. polon. sci., Classa sci. math. nat. Bi. (1-5B 1), 61.

DeLamater, E. D. (1950). The nuclear cytology of the vegetative diplophase of Saccharomyces cerevisiae. J. Bact. 60, 321.

FowELL, R. R. (1952). Sodium acetate agar as a sporulation medium for yeasts. Nature, Lond. 170, 578.

Fuhrmann, F. (1906). Die Kernteilung von Saccharomyces ellipsoideus. Zbl. Bakt., II, 15,769 . 
LEITz, K. (1951). Beitrag zur Hefecytologie. Arch. Mikrobiol., 16, 275.

Levan, A. (1947). Studies on the camphor reaction of yeast. Hereditas, 33, 431.

Lindegren, C. C. \& Lindegren, G. (1943). A new method for hybridizing yeast. Proc. Nat. Acad. Sci., Wash. 29, 306.

McClary, D. O., Nulty, W. L. \& Miller, G. R. (1959). Effect of potassium versus sodium in the sporulation of Saccharomyces. J. Bact. 78, 362.

McClary, D. O., Williams, M. A., Lindegren, C. C. \& OGur, M. (1957). Chromosome counts in a polyploid series of Saccharomyces. J. Bact. 73, 360.

Mortimer, R. K. (1958). Radiobiological and genetic studies on a polyploid series (haploid to hexaploid) of Saccharomyces cerevisiae. Radiation Research 9, 312.

Prahlada Rao, L. S. \& Subramaniam, M. K. (1952). Somatic chromosomal reduction in yeast. Arch. Mikrobol., 17, 160.

Roman, H., Phillips, M. M. \& Sands, S. M. (1955). Studies of polyploid Saccharomyces. I. Tetraploid segregation. Genetics, 40, 546.

Shinoto, Y. \& YUASA, A. (1941). Karyological studies in Saccharomyces cerevisiae. Cytologia, 11, 464 .

Subramaniam, M. K. (1946). Studies on the cytology of yeasts. I. Mitosis in Saccharomyces cerevisiae. Proc. Natn. Inst. Sci. India, 12, 143.

Swellengrebel, M. (1905). Sur la division nucleaire de la levure pressee. Ann. Inst. Pasteur, 19, 505.

Takahashi, T., SaIto, M. \& IKeda, Y. (1958). Heterothallic behaviour of a homothallic strain Saccharomyces yeast. Genetics, 43, 249.

YuASA, A. (1960). The spindle of the yeast-cell. Bot. Mag. Tokyo, 73, 474.

\section{EXPLANATION OF PLATES}

\section{Plate 1}

Figs. A-H. Meiosis in Saccharomyces cerevisiae, cross

Figs. A-C. These stages are considered to correspond to the prophase of meiosis.

Fig. A. The resting nucleus (Fig. $\mathbf{H}$ ) has started to divide.

Fig. B. Middle prophase; note clarified chromosome and chromatin.

Fig. C. Late prophase; some of the chromatin already coagulated and showing heteropycnosis.

Fig. D. Probably metaphase $I$, but showing considerable coagulation of chromatin; the number of chromatin masses was not constant from cell to cell.

Fig. E. Advanced stage of meiosis I; the chromatin coagulation has become more pronounced and one or more masses of chromatin are visible.

Fig. F. Tetrad.

Fig. G. Cells with diffuse nuclei; these cells were observed at almost equal frequencies in materials fixed after different times of growth on sporulating media (see Table 1). Accordingly, it seems reasonable to assume that these cells have no relation to spore formation.

Fig. H. Cell with resting nucleus.

Plate 2

Figs. A-L. Meiosis in Saccharomyces cerevisiae cross $\mathrm{H} 102 \times$ н 9 . All magnifications $\times 3600$

Figs. A-C. Stages of prophase of meiosis I.

Fig. C. Represents diakinesis.

Fig. D. Metaphase I with eighteen bivalents.

Fig. E. Anaphase I.

Fig. F. Metaphase II.

Fig. G. Anaphase II.

Fig. H. Telophase II.

Fig. I. Tetrad.

Figs. $J, K$ and $L$ are diagrammatic representations of photographs $C, D$ and $F$.

White rings of Fig. $\mathrm{L}$ represents unstained granules. 

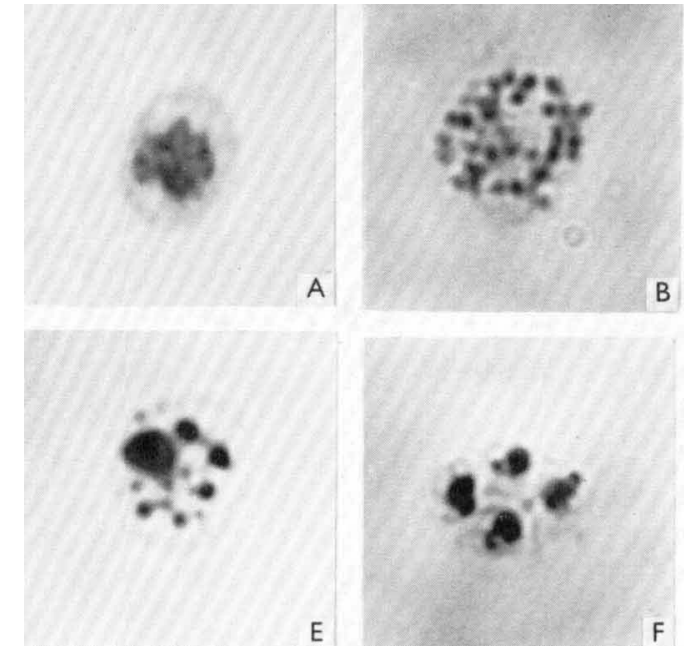
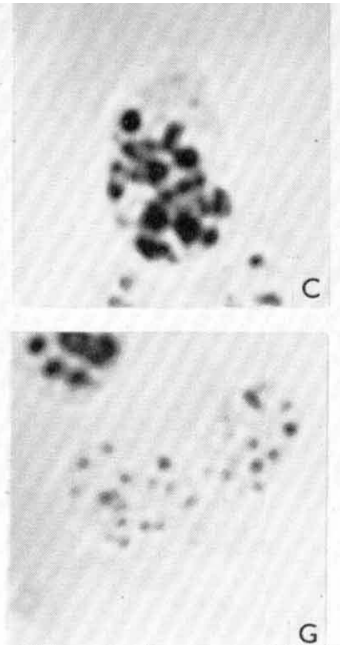
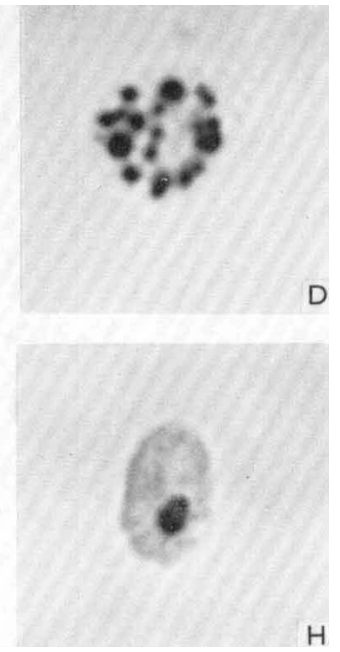

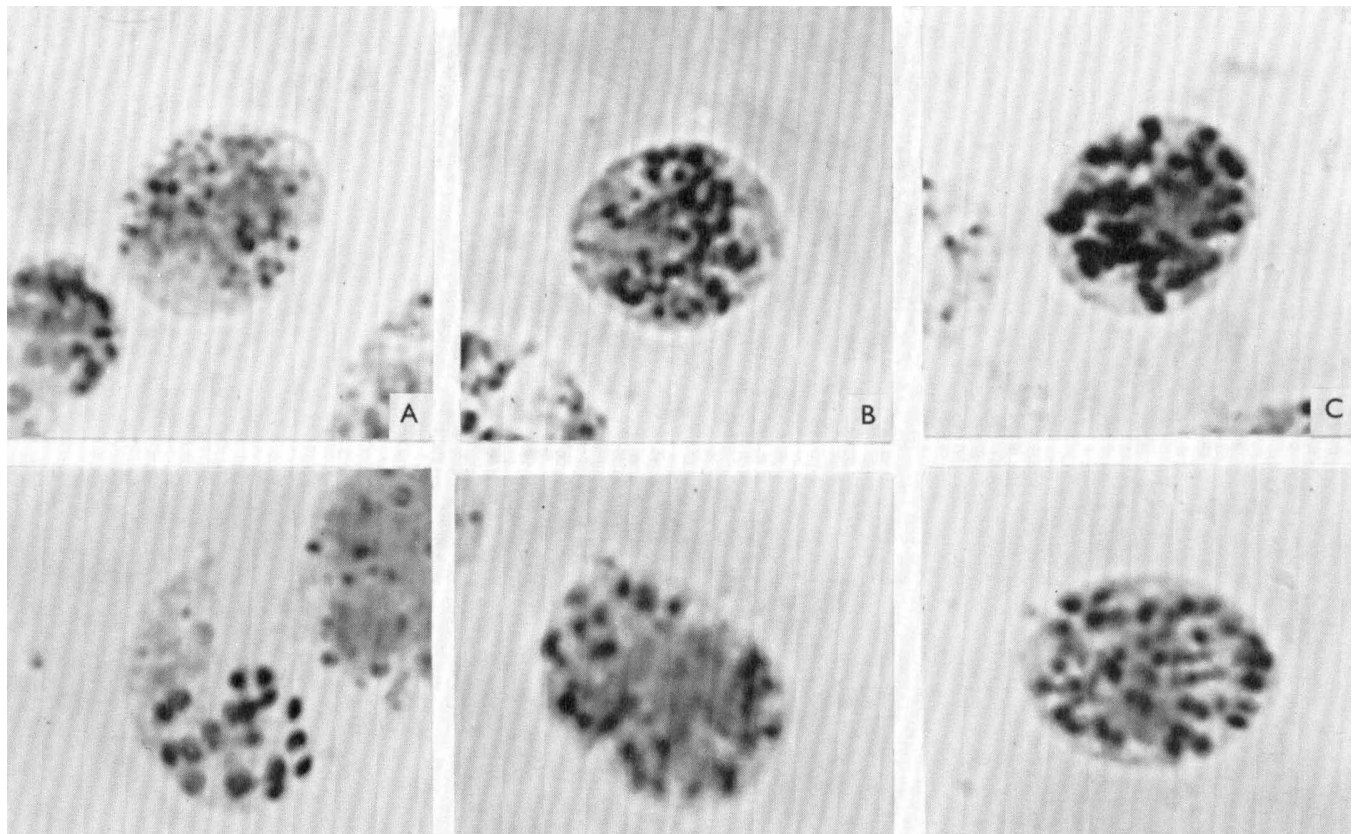

D

\section{E}
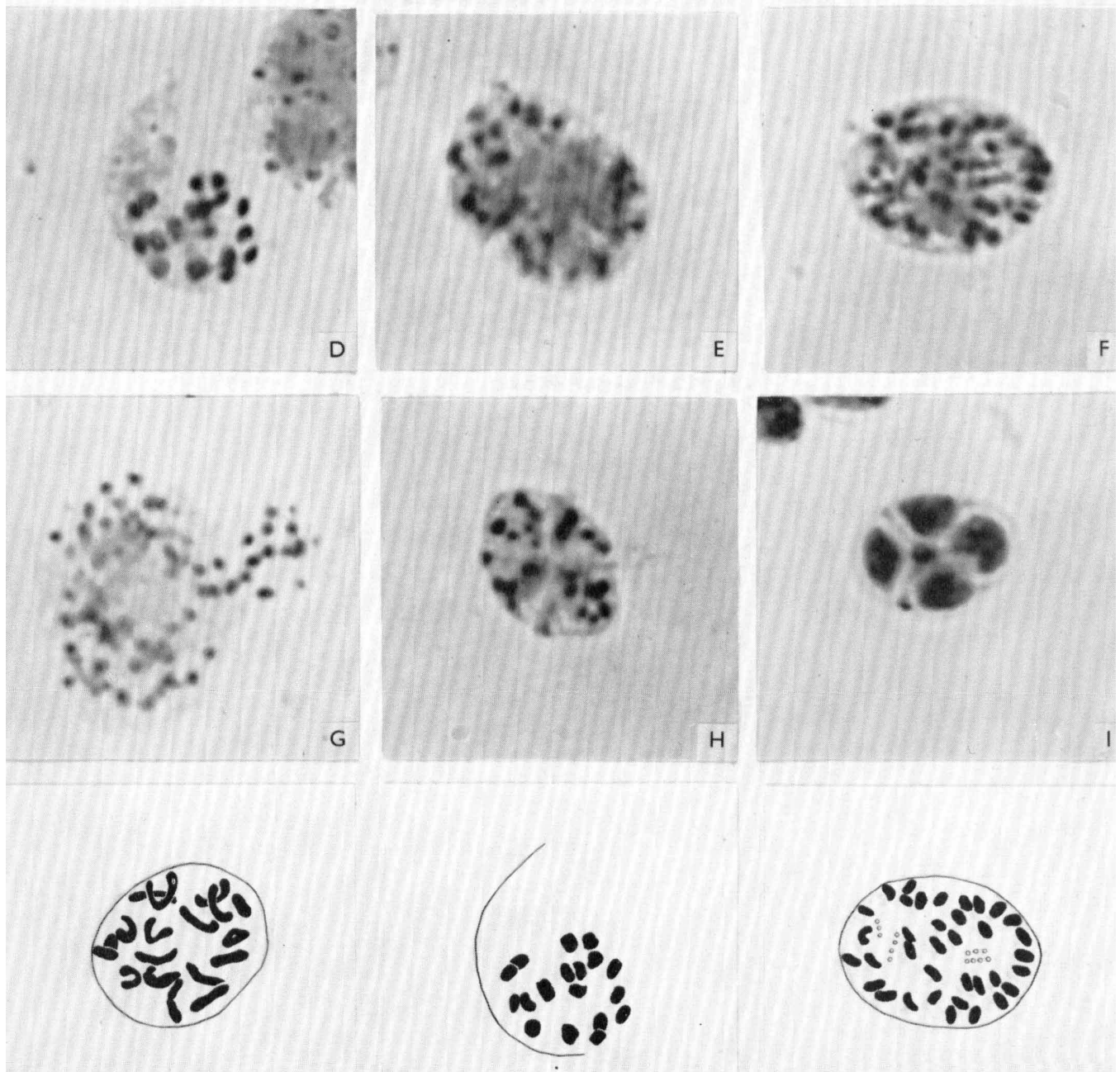

K

H. TAMAKI 